\title{
Intertube Excitonic Coupling in Nanotube Van der Waals Heterostructures
}

\author{
Maria G. Burdanova, Ming Liu, Michael Staniforth, Yongjia Zheng, Rong Xiang, \\ Shohei Chiashi, Anton Anisimov, Esko I. Kauppinen, Shigeo Maruyama, \\ and James Lloyd-Hughes*
}

Strong intertube excitonic coupling is demonstrated in 1D van der Waals heterostructures by examining the ultrafast response of radial C/BN/MoS core/shell/skin nanotubes to femtosecond infrared light pulses. Remarkably, infrared excitation of excitons in the semiconducting carbon nanotubes (CNTs) creates a prominent excitonic response in the visible range from the $\mathrm{MoS}_{2}$ skin, even with infrared photons at energies well below the bandgap of $\mathrm{MoS}_{2}$. Via classical analogies and a quantum model of the light-matter interaction these findings are assigned to intertube excitonic correlations. Dipole-dipole Coulomb interactions in the coherent regime produce intertube biexcitons, which persist for tens of femtoseconds, while on longer timescales (>100 ps) hole tunneling-from the CNT core, through the BN tunnel barrier, to the $\mathrm{MoS}_{2}$ skin-creates intertube excitons. Charge transfer and dipole-dipole interactions thus play prominent roles on different timescales, and establish new possibilities for the multi-functional use of these new nanoscale coaxial cables. by Moiré superlattice potentials. ${ }^{[4]}$ The diverse family of vdW heterostructures has been extended recently to include 1D vdW heterostructures, which resemble nanoscale coaxial cables, and which consist of radial heterojunctions of carbon nanotubes, insulating $\mathrm{BN}$ nanotubes and $\mathrm{MoS}_{2}$ nanotubes..$^{[5,6]}$ These functional nanocomposites show promise as nanoscale diodes ${ }^{[7]}$ and as photo-active nanocables ${ }^{[8]}$ for UV, visible, infrared, and $\mathrm{THz}$ optoelectronics. In order to develop these multi-functional materials it is vital to understand their fundamental photophysical properties, addressing questions such as: can charges transfer radially between different nanotube materials, and if so, how rapidly? And can manybody excitonic states exist across the heterojunctions of $1 \mathrm{D} \mathrm{vdW}$ materials?

\section{Introduction}

Atomically-thin semiconductors such as the transition metal dichalcogenides (TMDs) feature electron confinement in 2D and strong Coulomb interactions that produce large exciton binding energies. ${ }^{[1,2]}$ In 2D van der Waals (vdW) heterostructures dramatic modifications of the electronic and optical properties are known to arise, leading to unique effects like unconventional superconductivity ${ }^{[3]}$ and excitons confined
Before addressing these questions, we first set the scene by describing the current state-of-the-art in 2D vdW heterostructures. The Coulomb interaction between quasiparticles is known to play a prominent role in $2 \mathrm{D}$, for instance creating interlayer excitons, with an electron and a hole residing in different materials. ${ }^{[9-11]}$ Interlayer excitons are thought to form rapidly either via direct charge transfer or via Coulomb interactions that exchange energy between layers. With their larger polarizability and slower recombination than intralayer
M. G. Burdanova

Center for Photonics and 2D Materials

Moscow Institute of Physics and Technology (State University)

Institutsky Lane, 9, Dolgoprudny 141700, Russia

M. G. Burdanova, J. Lloyd-Hughes

University of Warwick

Department of Physics

Gibbet Hill Road, Coventry CV4 7AL, UK

E-mail: j.lloyd-hughes@warwick.ac.uk

The ORCID identification number(s) for the author(s) of this article can be found under https://doi.org/10.1002/adfm.202104969.

(C) 2021 The Authors. Advanced Functional Materials published by Wiley-VCH GmbH. This is an open access article under the terms of the Creative Commons Attribution License, which permits use, distribution and reproduction in any medium, provided the original work is properly cited.

DOI: $10.1002 / \mathrm{adfm} .202104969$

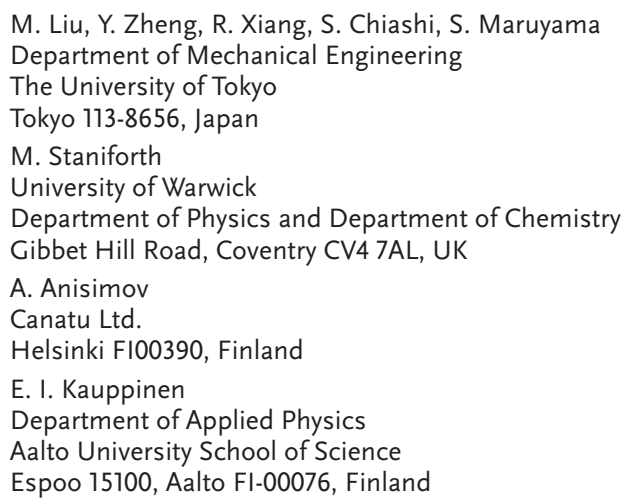


excitons, interlayer excitons provide a route to form free charges more efficiently in TMDs, ${ }^{[12]}$ and are hence better able to achieve the long-lived charges needed for photocatalysis and photoelectrochemistry, and have larger DC current flow, beneficial for optoelectronic devices such as photodiodes and photovoltaic cells. ${ }^{[13,14]}$

It can be challenging to study interlayer excitons in 2D materials via absorption spectroscopy: the transition rate to form interlayer excitons directly from light is relatively low, as the electron and hole are spatially separated. ${ }^{[1]}$ The ultrafast processes required to create interlayer excitons can, however, be studied by sensitive transient absorption spectroscopy methods, by first generating an intralayer exciton that subsequently dissociates across the junction, ${ }^{[15-18]}$ or by probing the internal transitions of interlayer excitons. ${ }^{[19]}$ In type II heterojunctions an electron-hole pair generated in one material will separate via charge transfer on ultrafast (sub-picosecond) timescales, creating a long-lived interlayer exciton that straddles the interface. ${ }^{[10]}$ Alternatively, Förster-type non-radiative dipole-dipole interactions can mediate rapid energy transfer between layers in vdW heterostructures, also on picosecond timescales. ${ }^{[20-22]}$ Later after photoexcitation, once a semiconductor's electrons have cooled by transferring energy to the lattice, ${ }^{[23]}$ heat transfer can further modify the electronic properties of adjacent layers and thereby change its absorption. Disentangling the combined effects of direct charge transfer, Coulomb-mediated energy transfer, and heat transport between layers in 2D vdW heterostructures is thus a significant challenge. ${ }^{[10]}$ It is therefore of fundamental and applied interest to examine charge transfer and energy coupling processes in 1D nanomaterials, where the Coulomb interaction has a different functional form than 2D, and to determine the strength or rate of each process.

\section{Results and Discussion}

In this contribution we report a significant intertube excitonic response for nano-coaxial cables formed from 1D vdW heterostructures (Figure 1a), which were fabricated by chemical vapor deposition on free-standing CNT template films. ${ }^{[5,6,8]}$ The cable's core consisted of bundles of semiconducting and metallic CNTs, which were wrapped by insulating BN nanotubes and an outer skin of semiconducting $\mathrm{MoS}_{2}$ nanotubes (NTs), with a $14.1 \mathrm{~nm}$ outer diameter and 2-3 $\mathrm{MoS}_{2}$ walls. ${ }^{[8]}$ The heteronanotubes were several microns long. The strong excitonic response of the CNTs and the $\mathrm{MoS}_{2}$ NTs at room temperature, combined with the long-range alignment of the different nanotubes within the heterostructure and large sample areas
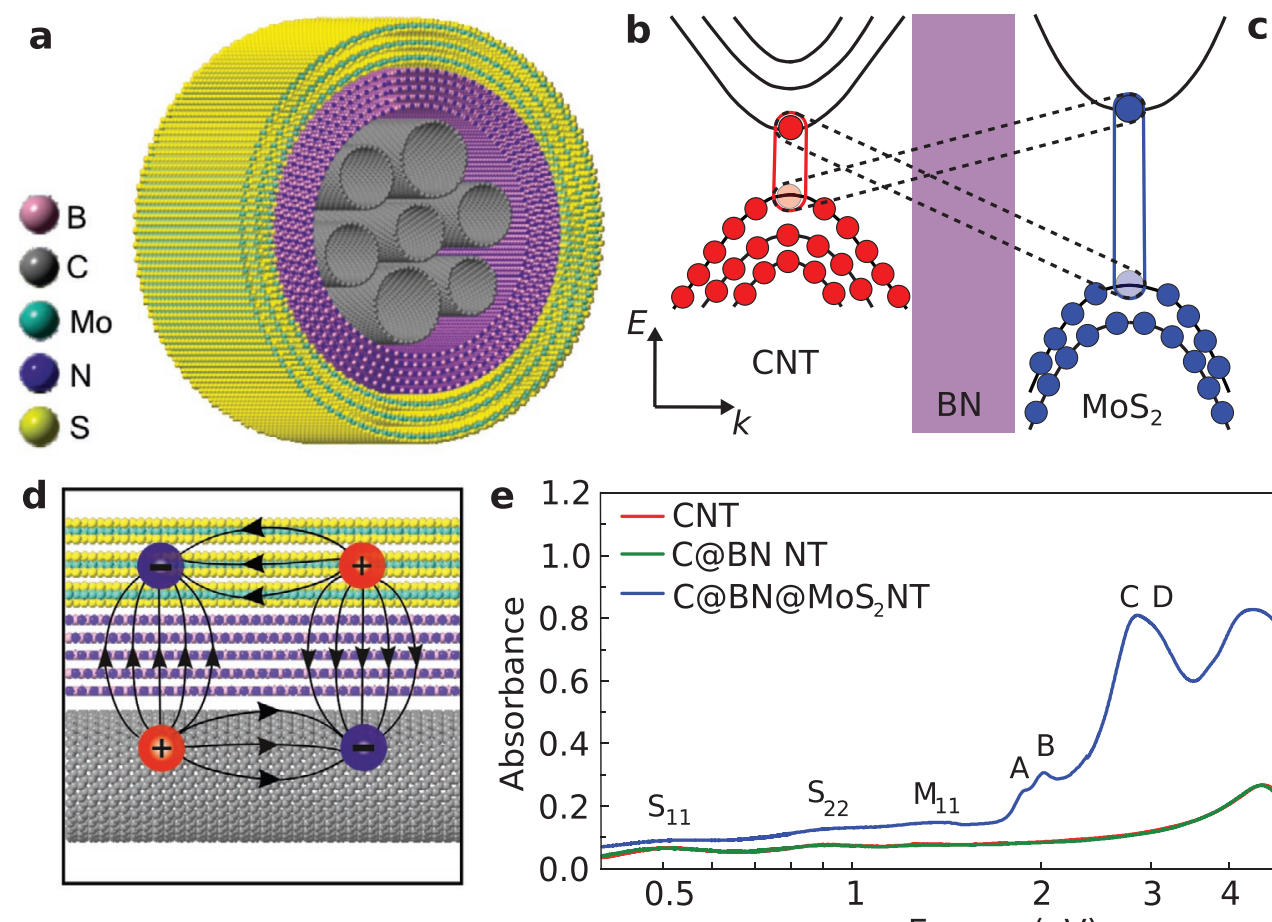
$\left(\approx 1 \mathrm{~cm}^{2}\right),{ }^{[5,8]}$ made this an ideal system to study the fundamentals of intertube electronic and excitonic coupling.

There are two distinct paradigms by which the light-matter interaction in our $1 \mathrm{D} \mathrm{vdW}$ heterostructures can be understood (Figure 1b). Either i) excitons (solid lines) behave independently of the quasiparticles in the other components of the heterostructure, and the optical properties are an effective medium average of the response of the independent constituents, or ii) the Coulomb forces between quasiparticles in different layers (dashed lines) create electronic correlations with a unique optical response. Simple physical arguments in favor of this second possibility can be advanced. For instance, electrostatics predicts that a free charge in a CNT will be screened by an opposite charge in the $\mathrm{MoS}_{2}$ skin (Figure 1c), forming an intertube exciton. A dipole field in the CNT, that is, an excitonic polarization, will induce a dipole in the $\mathrm{MoS}_{2}$ NT (Figure 1d), creating an intertube biexciton. If a coherent excitonic polarization is created in the CNT using an infrared pump pulse, one may predict that intertube biexcitons and intertube excitons form dynamically with different rates. An intertube biexciton should form rapidly, as the Coulomb interaction requires a negligible time to traverse the BN shell, while intertube excitons require longer to form as charge transfer between the CNT core and $\mathrm{MoS}_{2}$ skin must occur. By separating the core and skin with $\mathrm{BN}$ nanotubes charge transfer can be suppressed (BN's wide bandgap makes it an excellent tunnel barrier), slowing intertube excitonic formation.

Indirect evidence of the importance of intertube interactions comes from the optical absorption, Figure 1e, obtained for the pristine CNT films, after BN overgrowth, and after $\mathrm{MoS}_{2}$ nanotube growth. The C@BN@MoS heteronanotube films have an optical absorbance from the UV to the THz that can be roughly understood as resulting from the combination of the equilibrium absorbance of the constituent nanotubes in isolation. ${ }^{[8]}$ However, in the infrared range, below the band edge of the $\mathrm{MoS}_{2}$, the C@BN@MoS heteronanotube film has an enhanced absorbance in comparison to the C@BN and CNT reference films (Figure 1e). One explanation of this effect is that Coulomb correlations between the $\mathrm{MoS}_{2}$ and the CNTs enhances the excitonic absorption of the $S_{11}, S_{22}, M_{11}$ excitons in the CNTs, similar to the boost in absorption strength created by electron-hole pairs near the band edge of a direct gap semiconductor. Alternatively, the $\mathrm{MoS}_{2}$ growth may have modified the effective dielectric function, increasing the amount of reflection loss, or a fraction of the $\mathrm{MoS}_{2}$ nanotubes may be metallic ${ }^{[24]}$ and hence absorb in the infrared. Photoluminescence (PL) spectroscopy also provides indirect evidence of intertube interactions: for instance the A exciton's PL efficiency for C@BN@MoS NTs is lower than for BN@ MoS 2 NTs. ${ }^{[25]}$

In order to establish uniquely whether intertube Coulomb interactions modify the ground state electronic and the optical properties of $1 \mathrm{D} \mathrm{vdW}$ heterostructures, we introduce a scheme based on multi-color pump-probe spectroscopy (Figure 1f). A heterostructure with Coulomb-coupled states has excitonic energy levels $E_{|m n\rangle}$, where $m, n=0$ denotes the ground state, $|00\rangle$, $m=1$ denotes an $\mathrm{S}_{11}$ exciton in a semiconducting CNT, and $n=$ 1 labels an A or B exciton in the $\mathrm{MoS}_{2}$. By pumping at an energy $E_{1}=E_{|10\rangle}-E_{|00\rangle}$ in the infrared, a fraction of the system is moved from the ground state $|00\rangle$ to the $|10\rangle$ state. If the heterostructure has a common Coulomb-coupled ground state then an optical probe at $E_{2}=E_{|01\rangle}-E_{|00\rangle}$ exhibits a reduced absorption as a result of the lower ground state population. In contrast, if there are no Coulomb correlations between NTs, and the $\mathrm{MoS}_{2} \mathrm{NT}$ and CNT electronic ground states are therefore independent, then pumping at $E_{1}$ will not induce an absorption change at $E_{2}$ : the ground state population of the $\mathrm{MoS}_{2}$ NT will not be modified. By checking whether or not infrared excitation of $\mathrm{BN} @ \mathrm{MoS}_{2}$ NTs modifies the visible absorption, one can further test if $\mathrm{MoS}_{2} \mathrm{NTs}$ have any inherent absorption processes below the A exciton.

Ultrafast absorption spectroscopy experiments on thin films of C, C@BN, C@BN@MoS 2 and BN@MoS formed, pumping at $0.6 \mathrm{eV}$ (close to $E_{1}$ ) or at $3.0 \mathrm{eV}$, with a whitelight probe that covered the $\mathrm{A}(1.88 \mathrm{eV})$ and $\mathrm{B}(2.02 \mathrm{eV})$ excitons and interband absorption region of $\mathrm{MoS}_{2}$. The maps of the differential change in optical density, $\triangle \mathrm{OD}$, in Figure 2a demonstrate
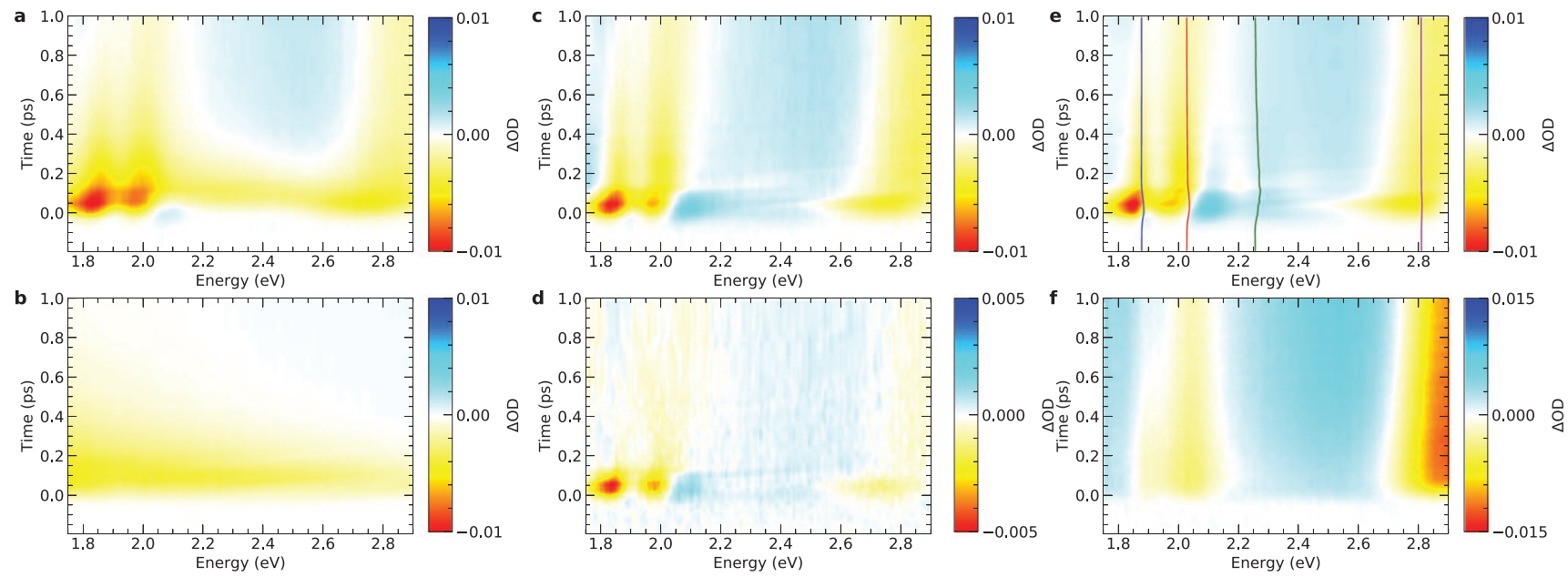

Figure 2. Transient absorption spectra at a range of pump-probe delay times for a) C@BN@MoS NTs and b) C@BN NTs, under excitation with a $0.62 \mathrm{eV}, 200 \mu \mathrm{J} \mathrm{cm}^{-2}, 35 \mathrm{fs}$ pump pulse. c) Data from (a) after subtracting the CNT response in (b). d) As (c), but for data obtained at pump fluence $70 \mu \mathrm{cm}^{-2}$. e) Multi-oscillator fit to the optical density under photoexcitation from (c). Vertical lines show the fitted oscillator energies versus time. f) Excitation with pump photons at $3.0 \mathrm{eV}$ produces changes dominated by the $\mathrm{A}, \mathrm{B}$, and interband absorption of the $\mathrm{MoS}_{2}$. 
that the $\mathrm{MoS}_{2}$ NTs' optical response is modified by the $0.6 \mathrm{eV}$ pump: for instance the $\mathrm{A}$ and $\mathrm{B}$ excitonic absorption peaks are suppressed (red areas are reduced optical density; higher transmission). As the pump photon energies $(0.6 \mathrm{eV})$ were only a third of the band edge of $\mathrm{MoS}_{2}(1.88 \mathrm{eV})$, and a $\mathrm{BN} @ \mathrm{MoS}_{2}$ sample prepared with the CNTs removed showed no discernible $\triangle \mathrm{OD}$ (Figures S1 and S2, Supporting Information), processes involving single-photon absorption by the $\mathrm{MoS}_{2}$ NTs (e.g., band-tail absorption; free-carrier absorption) can be ruled out. Nonlinear absorption processes such as three-photon absorption can also be eliminated by the lack of response from BN@MoS $\mathrm{M}_{2} \mathrm{NTs}$, further confirmed by the linearity of $\triangle \mathrm{OD}$ with pump fluence (Figure S3, Supporting Information). The modification of the excitonic response of the $\mathrm{MoS}_{2}$ can therefore be uniquely identified as resulting from coupling between the $S_{11}$ excitons generated in the CNTs and the electronic states of the $\mathrm{MoS}_{2}$.

To further elucidate the dynamic response of the C@BN@ $\mathrm{MoS}_{2}$ heteronanotubes, we performed reference experiments with a C@BN NT film without $\mathrm{MoS}_{2}$ NTs. Figure 2b shows a broadband, weaker response from the higher lying excitonic transitions in the CNTs (e.g., $\mathrm{S}_{33}, \mathrm{~S}_{44}$, and their continua). This transient response from the CNTs decays with lifetimes below 220 fs (Figure S4, Supporting Information). To examine the optical response of the $\mathrm{MoS}_{2}$ NTs without this contribution we subtracted the reference's $\triangle O D$ data set from that of the heteronanotubes, yielding Figure $2 c$, where features near zero pumpprobe delay can be seen more clearly. Using data obtained at lower fluence $\left(70 \mu \mathrm{J} \mathrm{cm}^{-2}\right)$, the same procedure yields Figure $2 \mathrm{~d}$. At early times and lower fluence the response from the $\mathrm{MoS}_{2}$ NTs persists for only $100 \mathrm{fs}$, with peaks in $\triangle \mathrm{OD}$ near the $\mathrm{A}$ and $\mathrm{B}$ excitons and $\mathrm{C}$ peak $(2.81 \mathrm{eV})$. The $\triangle \mathrm{OD}$ spectra at higher fluence are similar for the first $100 \mathrm{fs}$, whereupon a more persistent $\triangle \mathrm{OD}$ signal with a distinct spectral response appears.

Physically, the dynamics at early time result from the strong electric field of the IR pump pulse: this creates a coherent $S_{11}$ excitonic polarization in the CNTs, which then induces a polarization response from the $\mathrm{MoS}_{2}$ nanotubes via the Coulomb interaction, forming transient intertube biexcitonic states, as in
Figure 1d. The timescales for the transient rise and decay of this intertube exciton-exciton coupling were examined by fitting the experimental transmittance spectra at each pump-probe delay with a modeled transmittance calculated from a dielectric function built from multiple oscillators. To cover the experimental range of the transient absorption spectra, four oscillators were adopted (A and B excitons, interband transitions, and the $\mathrm{C}$ peak), and their center energy, oscillator strength, and linewidth were allowed to vary with delay. An example of the $\triangle \mathrm{OD}$ resulting from this fit is reported in Figure 2e, while the center energy and strength of the A exciton are reported in Figure 3 (full details of the fits and parameters are provided in the Figures S5-S7, Supporting Information). The dynamics following ultrafast excitation at $3.0 \mathrm{eV}$ were also measured (Figure 2f) and fitted: the band-edge excitonic response formed extremely rapidly, within $100 \mathrm{fs}$, in line with recent results on monolayer $\mathrm{MoS}_{2}$, and attributed to rapid exciton cooling mediated by the strong exciton-phonon interaction. ${ }^{[26]}$

Under IR excitation the A exciton's center energy undergoes a dynamic blueshift, evident in Figure 3a, which rises and decays with the IR laser pulse (shaded area, Gaussian pulse with $\sigma=35 \mathrm{fs}$ ). The peak blueshift increased with fluence. Similar blueshifts were seen for the B exciton and the interband oscillator at $2.25 \mathrm{eV}$ under IR excitation (Figure S7, Supporting Information), whereas direct excitation of the $\mathrm{MoS}_{2}$ NTs at $3.0 \mathrm{eV}$ produced a redshift in the A exciton energy as a result of bandgap renormalization. ${ }^{[27]}$ Within the coherent regime (during the IR pulse), the blueshift may be understood as an optical Stark shift of the exciton energies in the $\mathrm{MoS}_{2}$. Alternatively, with a finite population in state $E_{|10\rangle}$ there is an excited state absorption pathway (dashed line in Figure 1f) at an energy $E_{|11\rangle}-E_{|10\rangle}=E_{|01\rangle}-E_{|00\rangle}-\Delta$, that is, an energy $\Delta$ below the excitonic resonances of the $\mathrm{MoS}_{2}$ NTs. This excited state absorption could therefore counteract the ground state bleach of the $E_{|00\rangle}$ to $E_{|01\rangle}$ transition, if $\Delta$ is comparable to the linewidth of the excitonic absorption. As the magnitudes of the blueshifts $(<7 \mathrm{meV})$ are small compared to their linewidths $(>48 \mathrm{meV})$ we cannot conclusively identify their origin.
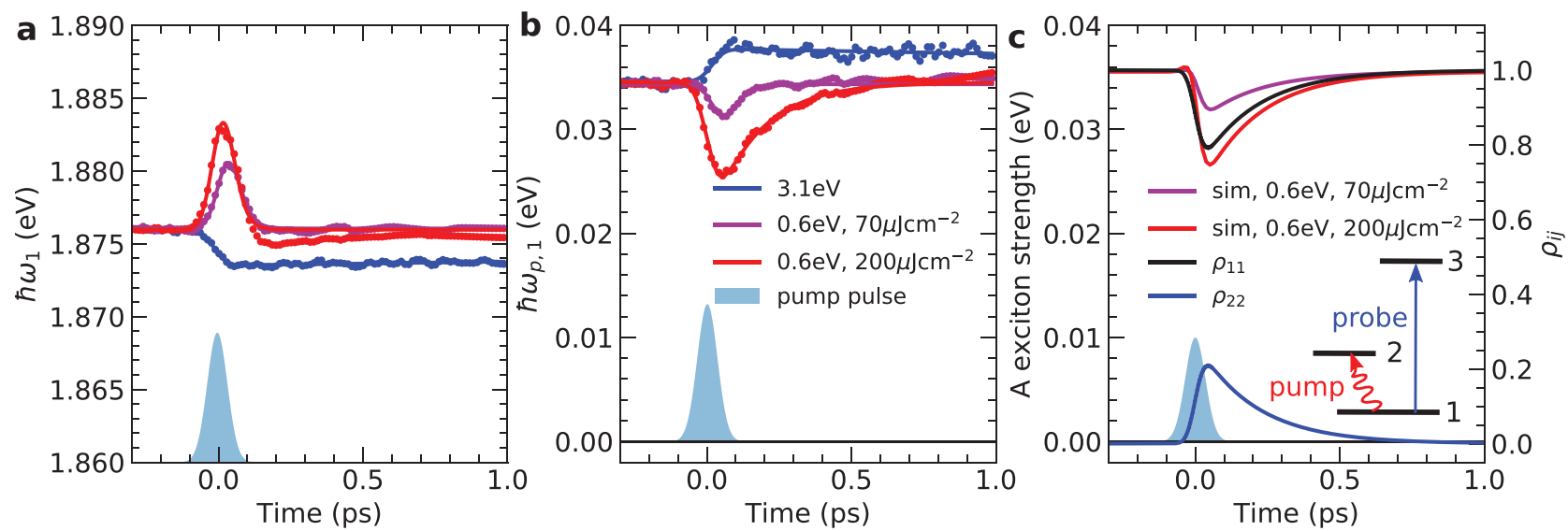

Figure 3. Time-dependent dynamics of the A exciton. a) The A exciton energy, $\hbar \omega_{1}$, blueshifts during the coherent regime only, with a larger blueshift for $200 \mu \mathrm{cm}^{-2}$ (red points) than $70 \mu \mathrm{cm}^{-2}$ (purple points). UV excitation produces a quasi-permanent redshift (blue points). Solid lines are fits using a Gaussian IR pulse envelope (shaded area, $\sigma=35 \mathrm{fs}$ ) and an exponential decay. b) As in (a), but for the A exciton's oscillator strength, $\hbar \omega_{p, 1}$. c) Bloch equation simulations of the ground state and CNT exciton populations, $\rho_{11}$ (black) and $\rho_{22}$ (blue), along with the simulated strength of the A exciton response at the same fluences as used in experiment (red and purple lines). 
The A oscillator strength (Figure 3b) lowered during the IR pump pulse, directly showing that fewer electrons were available to undergo the transitions that form A excitons. At times after the IR pump pulse the intertube biexcitonic response recovered rapidly toward equilibrium, with a time constant $\tau$ that was determined from fits to the dynamics (solid lines in Figure 3). At lower IR fluence we found $\tau=43 \mathrm{fs}$, suggesting the intertube biexcitonic response persisted essentially only within the coherent regime, while at higher IR fluence the intertube excitonic response was longer lived ( $\tau=166 \mathrm{fs}$ ). After the IR drive field has finished, the coherent $S_{11}$ polarization decoheres at a rate set by both exciton momentum scattering and exciton population transfer processes. For example, optically-bright $\mathrm{S}_{11}$ singlet excitons can relax to lower energy optically-dark triplet excitons. Alternatively, they can transfer their energy via nonradiative nearfield coupling to another nearby nanotube with a finite density of states at the same energy, ${ }^{[28]}$ such as a metallic CNT. Regardless of the mechanism by which the coherent $S_{11}$ excitonic polarization is removed, the induced excitonic response of the $\mathrm{MoS}_{2}$ is lowered when the $S_{11}$ polarization weakens. Decoherence of the excitonic polarization component in the $\mathrm{MoS}_{2}$ can also cause the destruction of intertube correlations. Hence the intertube biexcitonic contribution decays on timescales comparable to, or faster than, the timescale for recovery of the C@BN reference sample's interband response, which was 220 fs and faster (S4, Supporting Information). The intratube excitonic response of $\mathrm{A}$ and $\mathrm{B}$ excitons directly created in the $\mathrm{MoS}_{2}$ NTs under $3.0 \mathrm{eV}$ excitation recovered more slowly (Figure $2 \mathrm{f}$ ).

A full theoretical treatment of intertube excitonic coupling in the heterostructure requires the self-consistent inclusion of the semiconductor physics, for instance via the semi-classical semiconductor Bloch equations, along with consideration of the electromagnetism by solving Maxwell's equations. The current state-of-the-art in this area includes studies of superradiant effects in coupled 2D TMD layers ${ }^{[29]}$ and the rapid ultrafast formation dynamics of excitons in $\mathrm{MoS}_{2}$ monolayers after nonresonant visible excitation. ${ }^{[26]}$ Here we provide two simplified theoretical models that allow intuitive insights into intertube excitonic effects. First, a pair of coupled classical oscillators can represent the system: a first oscillator represents the excitonic polarization of an $\mathrm{S}_{11}$ exciton in a CNT, the second oscillator denotes an A exciton in a $\mathrm{MoS}_{2}$ NT, while a third spring models the Coulomb dipole-dipole coupling between excitons. When an impulse produces an excitonic polarization in the CNT oscillator (represented by the extension of the first spring from equilibrium), a damped oscillatory response from the $\mathrm{MoS}_{2}$ oscillator is then produced, with a spectral maximum close to the A exciton energy (Figure S8, Supporting Information).

Alternatively, a second theoretical model is to solve the Bloch equations in the excitonic basis for the lowest three levels in Figure 1f, as described in the Supporting Information. This approach improves on the classical model by including quantum effects such as finite level occupancy and dipole matrix elements, and includes electromagnetism self-consistently, but ignores many-body effects (e.g., bandgap renormalization) and the optical Stark effect, and includes dephasing and recombination only phenomenologically. Figure 3c illustrates the output from this simulation, with population decay times $\tau_{\mathrm{d}}=200 \mathrm{fs}$ and $60 \mathrm{ps}$ for $\mathrm{S}_{11}$ excitons (level 2) and $\mathrm{MoS}_{2}$
A excitons (level 3) respectively. During excitation of the $1 \rightarrow 2$ transition $\left(E_{|00\rangle}\right.$ to $E_{|10\rangle}$, shaded area), the rapid dephasing of the coherent excitonic response (represented by the components $\rho_{12}$ and $\rho_{21}$ of the density matrix) produced an $\mathrm{S}_{11}$ exciton population, $\rho_{22}$. The ground state population, $\rho_{11}$, and $\rho_{22}$ both returned toward equilibrium at a rate set by the fast population decay $\tau_{\mathrm{d}}$. Further, a full simulation of the pump-probe experiment was obtained using multiple Bloch simulations including a probe pulse resonant to the $1 \leftrightarrow 3$ transition and arriving at varying time delays. The dynamics of the pump-induced change in A oscillator strength from this model (red and purple lines in Figure $3 \mathrm{c}$ ) track the decay in $\mathrm{S}_{11}$ population, and are in excellent accord with the experimental data (Figure $3 \mathrm{~b}$ ).

This theoretical description provides further evidence that infrared excitation of excitons in the CNT core can yield strong modifications in the visible response of the $\mathrm{MoS}_{2}$ NT skin. Indeed, it is worth emphasizing the relative strength of the transient absorption: at comparable numbers of pump photons absorbed, the visible response induced by the IR pump is almost as strong as that induced by the UV pump (Figure 2).

Finally, we report the transient absorption dynamics of the heteronanotubes at later times after excitation. Under infrared excitation, the initial ultrafast response (discussed above) disappears within $1 \mathrm{ps}$, but is followed by a gradual enhancement of transient absorption over the first 1 ns, evident in Figure 4. This change is then long-lived, remaining constant until the end of the 3 ns experimental time window. Similar features were observed at different pump fluences, and were not observed on BN@ $\mathrm{MoS}_{2}$ or $\mathrm{C} @ \mathrm{BN}$ reference samples. This transient response indicates that a slower process, such as heat transport or charge transfer from the CNTs to the $\mathrm{MoS}_{2}$ NTs, has modified the absorption associated with the $\mathrm{A}$ and $\mathrm{B}$ exciton and interband absorption features of the $\mathrm{MoS}_{2}$ NTs. A photothermal explanation can be ruled out by examining the dynamics under UV excitation: under comparable pulse fluences UV and IR excitation would ultimately heat the NTs in a similar way and would thus be expected to produce the same long-lived response. However, excitation of the $\mathrm{MoS}_{2}$ NTs at $3.0 \mathrm{eV}$ led to a $\Delta \mathrm{OD}$ that reduced monotonically with time, with a characteristic lifetime of $60 \mathrm{ps}$ (Figure 4b,c) that was much faster than the long-lived feature under IR excitation, which persisted for $\gg 1 \mathrm{~ns}$.

Instead, the formation of indirect intertube excitons via charge transfer can account for the slow response under IR excitation. Indeed the characteristic timescale of $220 \mathrm{ps}$ for its rise (Figure 4c) is consistent with our estimates of hole tunneling times from the CNTs to the $\mathrm{MoS}_{2}$ : using a model of quantum tunneling through the $\mathrm{BN}$ barrier ${ }^{[30]}$ we estimated hole tunneling times of $100 \mathrm{ps}-1$ ns (Figure S9, Supporting Information). While the indirect excitons thus created do not directly absorb, the transfer of quasiparticles from one layer to another modifies the absorption rate and thus creates a nonzero $\triangle \mathrm{OD}$ in the same way as discussed for the ultrafast intertube biexcitonic response.

\section{Conclusion}

In summary, the results presented in this work show that intertube coupling plays a pivotal role in the optical response of 

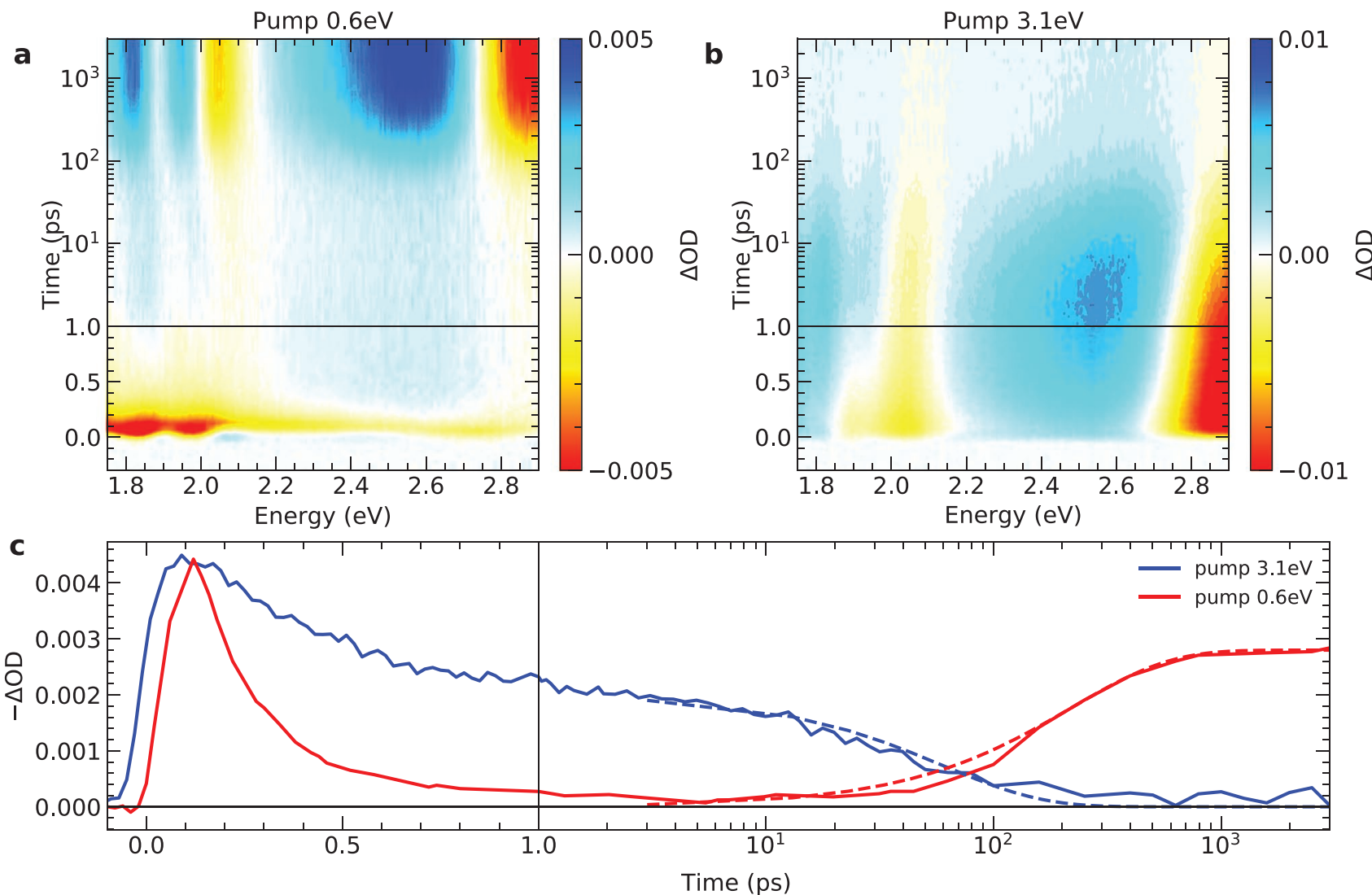

Figure 4. Time evolution of the pump induced change in OD for the C@BN@MoS ${ }_{2}$ heteronanotube film at later pump-probe delay times. a) Following IR excitation at $70 \mu \mathrm{cm}^{-2}$, the coherent intertube excitonic effects disappear within $1 \mathrm{ps}$, followed by a slower increase in the response from the $\mathrm{MoS}_{2}$, which then does not decay within the $3 \mathrm{~ns}$ time window of the experiment. b) Under direct (UV) excitation at $200 \mu \mathrm{J} \mathrm{cm}{ }^{-2}$ the transient OD recovers monotonically toward equilibrium. c) Slices at constant energy (averaged over 2.02-2.04 eV around the B exciton) show differing dynamics at later times for IR $(0.6 \mathrm{eV})$ and UV (3.1 eV) excitation. Simple fits (dashed lines) allow indicative timescales to be extracted: an exponential decay (of the form $y(t)=a e^{-t / \tau_{d}}$, with $\tau_{d}=60 \mathrm{ps}$ ) for direct excitation of the $\mathrm{MoS}_{2}$ at $3.0 \mathrm{eV}$, or an exponential rise (of the form $y(t)=a\left(1-e^{-t / \tau_{r}}\right.$ ), with $\tau_{r}=220 \mathrm{ps}$ ) for excitation at $0.6 \mathrm{eV}$.

quasi-1D van der Waals heterostructures. Infrared excitation of the carbon nanotube cores created a response from the $\mathrm{MoS}_{2}$ skin on two different timescales. The initial ultrafast response at early times (around $100 \mathrm{fs}$ ) was dominated by intertube biexcitons mediated by the Coulomb interaction between quasiparticles, while charge transfer via quantum tunneling at later times (around $100 \mathrm{ps)} \mathrm{produced} \mathrm{indirect} \mathrm{intertube} \mathrm{excitons.} \mathrm{The} \mathrm{results}$ demonstrate that the non-interacting, single exciton picture does not accurately describe the physics of such heterostructures, as the visible and UV optical properties derived from one component of a $1 \mathrm{D}$ vdW heterostructure (here the $\mathrm{MoS}_{2}$ NTs) can be manipulated by selective excitation of another constituent (the CNTs) at lower energies. While we have demonstrated efficient intertube coupling and the effective control of light at different wavelengths here for $1 \mathrm{D} \mathrm{vdW}$ heterostructures, our methodology and results are applicable more broadly to $2 \mathrm{D}$ heterostructures and may stimulate further work in this area. For instance, future work may look at whether long-lived intertube excitons may be more easily dissociated into free charges, allowing broadband (IR to UV) light harvesting to form mobile carriers. This makes these functional nano-coaxial cables particularly attractive for nanoscale photovoltaic or photocatalytic applications.

\section{Experimental Section}

Sample Preparation: Single-walled CNTs were synthesized via the floating-catalyst aerosol CVD method, ${ }^{[3]]}$ creating $20 \mathrm{~nm}$-thick films with a network comprising $10 \%$ individual and $90 \%$ bundled nanotubes $(\approx 7$ per bundle). A mean CNT diameter of $2.1 \mathrm{~nm}$ was determined from transmission electron microscopy on individual tubes, while tube lengths were above $10 \mu \mathrm{m} .{ }^{[8]}$ Free-standing CNT films were used as a matrix for $\mathrm{BN}$ and $\mathrm{MoS}_{2}$ NT growth by CVD. ${ }^{[5]}$ The pristine CNT film was preheated at $1050{ }^{\circ} \mathrm{C}$ in $\mathrm{Ar} / \mathrm{H}_{2}$ for $1 \mathrm{~h}$ at low pressure and then coated by BN NTs at $1050{ }^{\circ} \mathrm{C}(1-3 \mathrm{~h})$ at low pressure, and subsequently cooled slowly to room temperature. The BN NTs had a mean outer diameter of $11.1 \mathrm{~nm}$, with around $4 \mathrm{BN}$ walls. $\mathrm{MoS}_{2}$ growth was performed at $550{ }^{\circ} \mathrm{C}$ at low pressure, and also cooled slowly to room temperature, creating $\mathrm{MoS}_{2}$ NTs with $14.1 \mathrm{~nm}$ outer diameter (2-3 walls). Alternatively, to produce BN@MoS ${ }_{2}$ heterostructures without CNTs, a C@BN film was annealed under oxygen, leaving a BN NT template with the same dimensions as above, before subsequent growth of $\mathrm{MoS}_{2}$ NTs on the BN NT templates..$^{[5]}$

Spectroscopy: Absorbance spectra were obtained using a Lambda $1050 \mathrm{UV} / \mathrm{vis} / \mathrm{IR}$ spectrophotometer (200-1200 THz range; 250-1500 nm wavelength) and Bruker Vertex 70V IR and Perkin Elmer Spectrum GX FTIR spectrometers (3-200 THz). Raman spectra were obtained using a confocal microspectrometer (Labram, Jobin-Yvon Horiba) with 660 and $488 \mathrm{~nm}$ lasers (spectral resolution $0.5 \mathrm{~cm}^{-1}$ ) and Renishaw inVia Reflex Raman Microscopes with 442 and $532 \mathrm{~nm}$ lasers. 
Ultrafast Spectroscopy: The optical and IR pump beams were created by an optical parametric amplifier (TOPAS, Light Conversion), seeded with a $1 \mathrm{kHz}, 40 \mathrm{fs}, 800 \mathrm{~nm}$ pulse (from a Newport Spectra Physics Spitfire Ace) to create pulses with a tunable center wavelength. For the visible probe, a white-light supercontinuum was generated by focusing the attenuated $800 \mathrm{~nm}$ fundamental beam into a vertically translated $2 \mathrm{~mm}$-thick $\mathrm{CaF}_{2}$ window. Changes in absorbance created by the pump pulse ( $500 \mathrm{~Hz}$ modulation frequency) were measured through the change in transmitted probe intensity, using a fiber-coupled spectrometer. The experimental data were corrected numerically in the time-domain in order to account for the chirp of the white-light probe. Samples were measured in air at room temperature.

Rate Equation Model: To extract a time constant $\tau$ for a dynamical change in an oscillator's fit parameter, $\gamma(t)$, where $y$ could represent the oscillator strength (proportional to the population difference), linewidth, or center energy, the rate equation

$\frac{\mathrm{d} \gamma}{\mathrm{d} t}=A e^{-\left(t-t_{0}\right) / 2 \sigma^{2}}-\frac{\gamma}{\tau}$

was solved numerically for $\gamma(t)$. The first term represents a Gaussian generation rate to model the envelope of the pump pulse, centered at time $t_{0}$ and with width $\sigma$. A non-linear least-square curve fit yielded $\sigma$ and $\tau$.

\section{Supporting Information}

Supporting Information is available from the Wiley Online Library or from the author.

\section{Acknowledgements}

M.G.B. acknowledges support from the Global Education Program (Russia) for a Ph.D. scholarship. The UK authors acknowledge the EPSRC (UK) for funding under grant EP/N010825/1. Part of this work was supported by JSPS KAKENHI (grant numbers JP18H05329, JP19H02543, JP2OH00220, and JP20KK0114) and by JST, CREST grant number JPMJCR20B5, Japan.

\section{Conflict of Interest}

The authors declare no conflict of interest.

\section{Data Availability Statement}

The data that support the findings of this study are available from the corresponding author upon reasonable request.

\section{Keywords}

excitons, nanotubes, ultrafast spectroscopy, van der Waals heterostructures

Received: May 25, 2021

Revised: August 18, 2021

Published online:

[1] S. Tongay, J. Suh, C. Ataca, W. Fan, A. Luce, J. Kang, J. Liu, C. Ko, R. Raghunathanan, J. Zhou, F. Ogletree, J. Li, J. Grossman, J. Wu, Scientific Reports 2013, 3, 2657.
[2] A. Chernikov, A. M. van der Zande, H. M. Hill, A. F. Rigosi, A. Velauthapillai, J. Hone, T. F. Heinz, Phys. Rev. Lett. 2015, 115, 126802.

[3] Y. Cao, V. Fatemi, S. Fang, K. Watanabe, T. Taniguchi, E. Kaxiras, P. Jarillo-Herrero, Nature 2018, 556, 43.

[4] K. Tran, G. Moody, F. Wu, X. Lu, J. Choi, K. Kim, A. Rai, D. A. Sanchez, J. Quan, A. Singh, J. Embley, A. Zepeda, M. Campbell, T. Autry, T. Taniguchi, K. Watanabe, N. Lu, S. K. Banerjee, K. L. Silverman, S. Kim, E. Tutuc, L. Yang, A. H. MacDonald, X. Li, Nature 2019, 567, 71.

[5] R. Xiang, T. Inoue, Y. Zheng, A. Kumamoto, Y. Qian, Y. Sato, M. Liu, D. Tang, D. Gokhale, J. Guo, K. Hisama, S. Yotsumoto, T. Ogamoto, H. Arai, Y. Kobayashi, H. Zhang, B. Hou, A. Anisimov, M. Maruyama, Y. Miyata, S. Okada, S. Chiashi, Y. Li, J. Kong, E. I. Kauppinen, Y. Ikuhara, K. Suenaga, S. Maruyama, Science 2020, 367, 537.

[6] R. Xiang, S. Maruyama, Small Sci. 2021, 1, 2000039.

[7] Y. Feng, H. Li, T. Inoue, S. Chiashi, S. V. Rotkin, R. Xiang, S. Maruyama, ACS Nano 2021, 15, 5600.

[8] M. G. Burdanova, R. J. Kashtiban, Y. Zheng, R. Xiang, S. Chiashi, J. M. Woolley, M. Staniforth, E. Sakamoto-Rablah, X. Xie, M. Broome, J. Sloan, A. Anisimov, E. I. Kauppinen, S. Maruyama, J. Lloyd-Hughes, Nano Lett. 2020, 20, 3560.

[9] P. Rivera, J. R. Schaibley, A. M. Jones, J. S. Ross, S. Wu, G. Aivazian, P. Klement, K. Seyler, G. Clark, N. J. Ghimire, J. Yan, D. G. Mandrus, W. Yao, X. Xu, Nat. Commun. 2015, 6, 6242.

[10] C. Jin, E. Y. Ma, O. Karni, E. C. Regan, F. Wang, T. F. Heinz, Nat. Nanotechnol. 2018, 13, 994.

[11] P. Rivera, H. Yu, K. L. Seyler, N. P. Wilson, W. Yao, X. Xu, Nat. Nanotechnol. 2018, 13, 1004.

[12] H. C. Kamban, T. G. Pedersen, Sci. Rep. 2020, 10, 5537.

[13] M.-L. Tsai, S.-H. Su, J.-K. Chang, D.-S. Tsai, C.-H. Chen, C.-I. Wu, L.-J. Li, L.-J. Chen, J.-H. He, ACS Nano 2014, 8, 8317.

[14] L. Hao, Y. Liu, W. Gao, Z. Han, Q. Xue, H. Zeng, Z. Wu, J. Zhu, W. Zhang, J. Appl. Phys. 2015, 117, 114502.

[15] K. Wang, B. Huang, M. Tian, F. Ceballos, M.-W. Lin, M. MahjouriSamani, A. Boulesbaa, A. A. Puretzky, C. M. Rouleau, M. Yoon, H. Zhao, K. Xiao, G. Duscher, D. B. Geohegan, ACS Nano 2016, 10, 6612.

[16] X. Wang, K. Shinokita, H. E. Lim, N. B. Mohamed, Y. Miyauchi, N. T. Cuong, S. Okada, K. Matsuda, Adv. Funct. Mater. 2018, 29, 1806169 .

[17] C. Jin, E. C. Regan, D. Wang, M. I. B. Utama, C.-S. Yang, J. Cain, Y. Qin, Y. Shen, Z. Zheng, K. Watanabe, T. Taniguchi, S. Tongay, A. Zettl, F. Wang, Nat. Phys. 2019, 15, 1140.

[18] S. Sim, D. Lee, J. Lee, M. Cha, S. Cha, W. Heo, S. Cho, W. Shim, K. Lee, J. Yoo, R. P. Prasankumar, H. Choi, M.-H. Jo, Phys. Rev. B 2020, 101, 17.

[19] P. Merkl, F. Mooshammer, P. Steinleitner, A. Girnghuber, K. Q. Lin, P. Nagler, J. Holler, C. Schüller, J. M. Lupton, T. Korn, S. Ovesen, S. Brem, E. Malic, R. Huber 2019, 18, 691.

[20] H. Qian, C. Georgi, N. Anderson, A. A. Green, M. C. Hersam, L. Novotny, A. Hartschuh, Nano Lett. 2008, 8, 1363.

[21] D. Kozawa, A. Carvalho, I. Verzhbitskiy, F. Giustiniano, Y. Miyauchi, S. Mouri, A. H. Castro Neto, K. Matsuda, G. Eda, Nano Lett. 2016, 16, 4087.

[22] M. Selig, E. Malic, K. J. Ahn, N. Koch, A. Knorr, Phys. Rev. B 2019, 99, 035420

[23] M. Monti, K. D. G. I. Jayawardena, E. Butler-Caddle, R. M. I. Bandara, J. M. Woolley, M. Staniforth, S. R. P. Silva, J. LloydHughes, Phys. Rev. B 2020, 102, 245204.

[24] I. Milošević, B. c. v. Nikolić, E. Dobardžć, M. Damnjanović, I. Popov, G. Seifert, Phys. Rev. B 2007, 76, 233414.

[25] M. Liu, K. Hisama, Y. Zheng, M. Maruyama, S. Seo, A. Anisimov, T. Inoue, E. I. Kauppinen, S. Okada, S. Chiashi, R. Xiang, S. Maruyama, ACS Nano 2021, 15, 8418. 
[26] C. Trovatello, F. Katsch, N. J. Borys, M. Selig, K. Yao, R. BorregoVarillas, F. Scotognella, I. Kriegel, A. Yan, A. Zettl, P. J. Schuck, A. Knorr, G. Cerullo, S. D. Conte, Nat. Commun. 2020, 11, 5277.

[27] C. Zhu, Y. Liu, J. Xu, Z. Nie, Y. Li, Y. Xu, R. Zhang, F. Wang, Sci. Rep. 2017, 7, 11221.

[28] R. D. Mehlenbacher, T. J. McDonough, M. Grechko, M.-Y. Wu, M. S. Arnold, M. T. Zanni, Nat. Commun. 2015, 6, 6732.
[29] C. E. Stevens, T. Stroucken, A. V. Stier, J. Paul, H. Zhang, P. Dey, S. A. Crooker, S. W. Koch, D. Karaiskaj, Optica 2018, 5, 749.

[30] D. Yadav, M. Trushin, F. Pauly, Phys. Rev. Res. 2020, 2, 043051.

[31] A. G. Nasibulin, A. Moisala, D. P. Brown, H. Jiang, E. I. Kauppinen, Chem. Phys. Lett. 2005, 402, 227. 